\title{
Flare Activity and Cluster Membership Probability of Flare Stars
}

\author{
L.V. Mirzoyan, V.V. Hambarian, A.L. Mirzoyan \\ Byurakan Astrophysical Observatory, Armenia
}

Mirzoyan (1976) showed that the concentration of flare stars around the center of the Pleiades cluster (Alcyone) was the same, irrespective of their proper motions. At that time, however, proper motions of only a few flare stars were known. The result was confirmed on the basis of more extensive observational material (Chavushian 1979, Mirzoyan 1983). Photographic observations of stellar flares in the general galactic field during 181 hours yielded the detection of only a single flare, i.e. the percentage of flare stars in the general galactic field is about $10 \%$ of the total number of flare stars detected in the regions of stellar clusters and associations (Chavushian 1979, Mirzoyan et al. 1988).

This result shows that flare activity can be considered as a definitive cluster membership criterion, which appears to be a better one than the cluster membership probability, which is based on proper motions. To prove this, cluster membership probabilities (Stauffer et al. 1991) for 408 Pleiades cluster flare stars from the catalogue by Haro et al. (1982), are used (Table 1).

Table 1. The distribution of the cluster membership probabilities $P$ for Pleiades flare stars according to Stauffer et al. (1991)

\begin{tabular}{cc}
\hline$P$ & Number of stars \\
\hline $0.0-0.2$ & 193 \\
$0.2-0.4$ & 14 \\
$0.4-0.5$ & 17 \\
$0.5-0.6$ & 13 \\
$0.6-0.8$ & 56 \\
$0.8-1.0$ & 135 \\
\hline Total & 408 \\
\hline
\end{tabular}

All flare stars of Table 1 were divided into two groups: those with $P>0.5$, being probable members of the Pleiades cluster, and those with $P<0.5$, probable non-members. A plot of their surface distributions around the centre of the Pleiades shows the absence of essential differences between both distributions, nor are any differences seen between the distributions of surface stellar density $d(r)$ around the centre of the Pleiades, when weights $P$ and $(1-P)$ are taken (Mirzoyan et al. 1993). Hence, the new cluster membership probabilities of flare stars based on proper motions confirm the conclusion that the overwhelming 
majority of flare stars is connected with the Pleiades cluster.

On the other hand, the inspection of the new cluster membership probabilities indicates that the concentration of flare stars around the center of the Pleiades is stronger for flare stars of comparatively high luminosities: almost all flare stars of comparatively high luminosities belong to the cluster. Such a regularity is also observed for the flare stars of the Orion association (Mirzoyan et al. 1993). Therefore it can be supposed that all flare stars of comparatively high luminosities are members of star clusters and associations, i.e. they are formed in these stellar systems and at present, the further they are from their centres, the lower are their luminosities (smaller masses).

The detection of flare activity, especially for stars with small flare frequencies, is very difficult. For practical purposes, it is convenient to use another criterion which can be determined more easily. The presence of strong emission lines in the spectra of flare stars is such a criterion.

Almost all spectroscopically studied flare stars of the Pleiades region have rather intense emission lines of $\mathrm{H}$ and CaII (e.g. Stauffer 1980, Mirzoyan et al. 1990). In their spectral study of the Hyades and Pleiades red dwarfs, Kraft \& Greenstein (1969) found that the existence of strong $\mathrm{H}$ and $\mathrm{Ca} I \mathrm{I}$ emission can be considered as a powerful criterion for cluster membership.

Prosser at al. (1991) showed that there is a substantial difference in $\mathrm{H} \alpha$ emission line strength between flare and non-flare stars in the Pleiades. A few exceptions from this rule can be explained by the fact that all low luminosity stars are flare stars, but not all of them are yet known as flare stars (Ambartsumian 1969). Hence, on the basis of existing observational data on flare stars, the flare activity can be considered as a powerful criterion for their membership in nearby clusters and associations.

\section{References}

Ambartsumian V.A., 1969, in Stars, Nebulae, Galaxies, V.V. Sobolev (ed.), Acad. Sci. of Armenia, Yerevan, p. 283

Chavushian H.S., 1979, A Study of Flare Stars in the Region of the Pleiades Aggregate, Byurakan Astrophys. Obs.

Haro G., Chavira E., Gonzalez G., 1982, Bol. Inst. Tonantzintla 3, No. 1

Kraft R.P., Greenstein J.L., 1969, in Low Luminosity Stars, S.S. Kumar (ed.), Gordon and Breach, New York, p. 65

Mirzoyan L.V., 1976, in Stars and Galaxies from Observational Points of View, E.K. Kharadse (ed.), Georgian Acad. Sci., Tbilisi, p. 121

Mirzoyan L.V., 1983, Astrofiz. 18, 588

Mirzoyan L.V., Hambarian V.V., Garibjanian A.T., Mirzoyan A.L., 1988, Astrofiz. 29, 531

Mirzoyan L.V., Hambarian V.V., Mirzoyan A.L., 1993, Astrofiz. 36, No. 3

Mirzoyan L.V., Hambarian V.V., Garibjanian A.T., Mirzoyan A.L., 1990, Astrofiz. 33, 5

Prosser C.F., Stauffer J.R., Kraft R.P., 1991, AJ 101, 1361

Stauffer J.R., 1980, AJ 85, 1341

Stauffer J.R., Klemola A., Prosser C., Probst R., 1991, AJ 101, 980 